\title{
Surgical Myectomy after Failed Ablation for Hypertrophic Obstructive Cardiomyopathy
}

\author{
loannis Bougioukas ${ }^{1} \quad$ Uta Hoppe $^{2} \quad$ Bernhard Danner $^{1} \quad$ Friedrich A. Schoendube $^{1}$ \\ ${ }^{1}$ Department of Thoracic and Cardiovascular Surgery, Georg-Agust \\ University, Goettingen, Germany \\ 2 Department of Cardiology, Paracelsus Medical University, Salzburg, \\ Austria \\ Address for correspondence Dr. med. Ioannis Bougioukas, \\ Universitatsklinikum Gottingen, Robert-Koch Str. 40, Goettingen \\ 37075, Germany (e-mail: ybougioukas@yahoo.com). \\ Thorac Cardiovasc Surg Rep 2016;5:30-32.
}

\author{
Abstract \\ Keywords \\ - hypertrophic \\ obstructive \\ cardiomyopathy \\ - septal myectomy \\ - alcohol septal \\ ablation
}

Background Hypertrophic cardiomyopathy is a genetic disease of the myocardial sarcolemma characterized by left ventricular hypertrophy. When obstruction to the left ventricular outflow tract is present and symptoms are refractory to medication, surgical myectomy or alcohol septal ablation is indicated.

Case Description We report a case of a patient presented for myectomy due to recurrence only 1 year after alcohol ablation. Interesting findings were a firm subaortic membrane and a direct insertion of the papillary muscle into the mitral valve.

Conclusion After myectomy and extensive papillary muscle mobilization, a significant relief of obstruction was achieved.

\section{Introduction}

Hypertrophic cardiomyopathy (HCM) is a genetic disease with congenital or spontaneous mutations of different proteins of the sarcolemma. It is characterized by mostly asymmetric left ventricular hypertrophy associated with myocardial disarray. The presence of obstruction to the left ventricular outflow tract (LVOT) with pressure gradients of $\geq 50 \mathrm{~mm} \mathrm{Hg}$ at rest or after provocation specifies the disease as obstructive HCM (HOCM). ${ }^{1}$

Treatment of HCM is based on medical therapy, but in HOCM with accompanied refractory symptoms, such as syncopes, dyspnea, and angina, surgical myectomy or alcohol septal ablation (ASA) is indicated. ${ }^{2}$ During the past years, surgery has been the gold standard for symptomatic patients, but with introduction of catheter-based occlusion of the first septal branch leading to a local myocardial infarction and reduction in the septal mass, a tendency toward the latter method has been established. ${ }^{3}$ Although some clinicians strongly suggest alcohol ablation as the treatment of choice for patients with symptomatic HOCM, a controversy still remains whether ASA could displace myectomy as the gold standard.

received

December 16, 2015

accepted

February 8, 2016

published online

April 4, 2016
DOI http://dx.doi.org/ 10.1055/s-0036-1580601. ISSN 2194-7635.
We present a case of a patient with HOCM referred to us for surgical myectomy only 1 year after having ASA. Interesting intraoperative findings were a firm subaortic membrane and a fixation of the posterior papillary muscle on the anterior mitral valve leaflet, both contributing to obstruction and mitral valve regurgitation.

\section{Case Presentation}

A 58-year-old female patient was referred to our department with diagnosis of hypertrophic obstructive cardiomyopathy. The patient had already been subjected to ASA 1 year ago. Peak-to-peak and LVOT pressure gradients were 16 and $18 \mathrm{~mm}$ $\mathrm{Hg}$ at rest and 122 and $81 \mathrm{~mm} \mathrm{Hg}$ after provocation, respectively, before ASA. Occlusion of the septal branch was performed with injection of $96 \%$ ethanol and the postintervention course of the patient was uneventful, except a left anterior hemiblock. After intervention, peak-to-peak and LVOT pressure gradients of 7 and $13 \mathrm{~mm} \mathrm{Hg}$ were documented at rest, respectively. One-year follow-up echocardiographic study of the patient revealed recurrent LVOT obstruction with a $68-\mathrm{mm}$ $\mathrm{Hg}$ gradient at rest $\left(\mathrm{V}_{\max } 4.1 \mathrm{~m} / \mathrm{s}\right)$, while midventricular gradient was measured to be $80 \mathrm{~mm} \mathrm{Hg}\left(\mathrm{V}_{\max } 4.4 \mathrm{~m} / \mathrm{s}\right)$. A
License terms

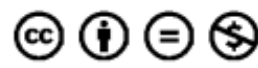

(c) 2016 Georg Thieme Verlag KG Stuttgart · New York 
systolic anterior motion (SAM) of the anterior mitral valve leaflet with moderate to severe mitral valve regurgitation was demonstrated in both echocardiography and magnetic resonance imaging. The presyncopes of the patient were attributed to the recurrence of HOCM, as an implanted event recorder did not show any arrhythmogenic abnormalities. Coronary angiography excluded coronary disease.

The operation was performed through conventional sternotomy. At the time of operation, transesophageal echocardiography showed in LVOT a $\mathrm{V}_{\max }$ of $7.35 \mathrm{~m} / \mathrm{s}$ (-Fig. 1) and a septum thickness of $24 \mathrm{~mm}$. The ascending aorta and both venae cavae were cannulated. Cardioplegia was administrated both antegrade and retrograde, and the procedure was performed in normothermia. After aortotomy, the aortic cusps were pulled up to gain access to the LVOT. The first interesting finding was a circular membrane fixed with hypertrophied trabeculae to the midventricular part of the LVOT. Additionally, a part of the posterior papillary muscle was fused within the anterior mitral leaflet, representing a mechanical substrate for the midventricular obstruction (-Fig. 2). The mitral valve itself was free of any deficiency. At first, the circular membrane including the inserting trabeculae was completely resected. The fused part of the papillary muscle was then resected, leading to a complete mobilization of the papillary muscle and to an extended relief of the left ventricle obstruction. Despite these maneuvers, a subaortic eccentric hypertrophy still remained, so that a typical extended myectomy was also performed. After removing the aortic clamp, complete atrioventricular block (AV-block) necessitated DDD-pacemaker stimulation. Because of persistence of the AV-block, a permanent pacemaker was implanted on the seventh postoperative day and the patient was then discharged from our institution. The postoperative echocardiography showed a very good systolic function of the LV and a $\mathrm{V}_{\max }$ in LVOT of $1.68 \mathrm{~m} / \mathrm{s}$, and pressure gradient of $12 \mathrm{~mm} \mathrm{Hg}$. No SAM was noted, and both aortic and mitral valves were fully competent.

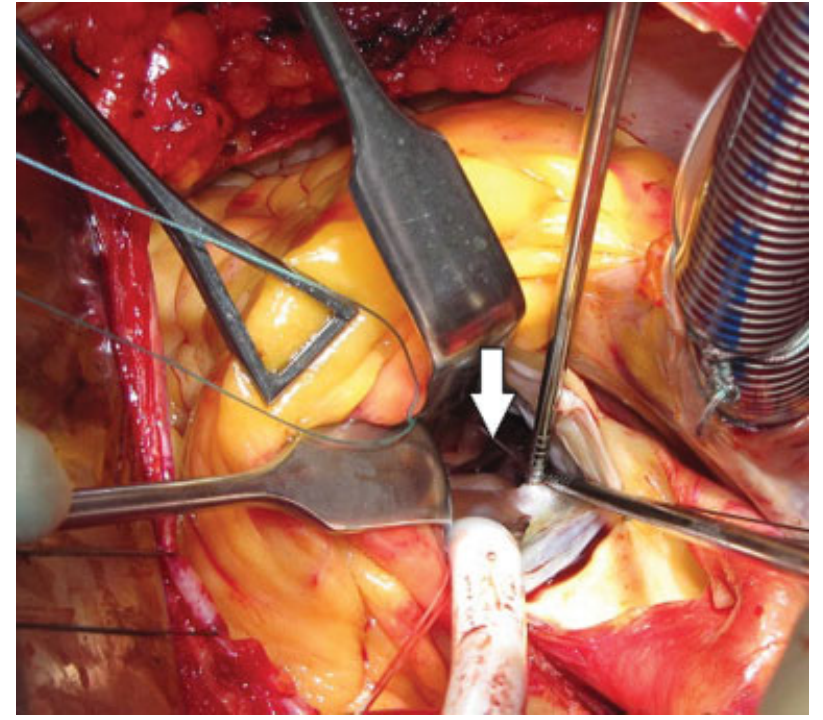

Fig. 2 View of the obstructive LVOT after the aortic cusps have been pulled up. The arrow shows the fixed insertion of the papillary muscle into the anterior mitral leaflet.

\section{Discussion}

Surgical myectomy has been considered to be the gold standard treatment approach for symptomatic patients with HOCM. In the hands of an experienced surgeon, surgical treatment of HOCM offers excellent results in terms of symptom relief, with a minimal rate of postoperative complications, such as complete heart block, injury to aortic and mitral valves requiring repair or even replacement, or ventricular septal defect. ${ }^{2,4}$ The introduction of alcohol ablation of the septum through occlusion of the first septal branch of the left anterior descending artery via catheter approach had managed to gain significant support from the cardiologic society, mainly because of the minimal

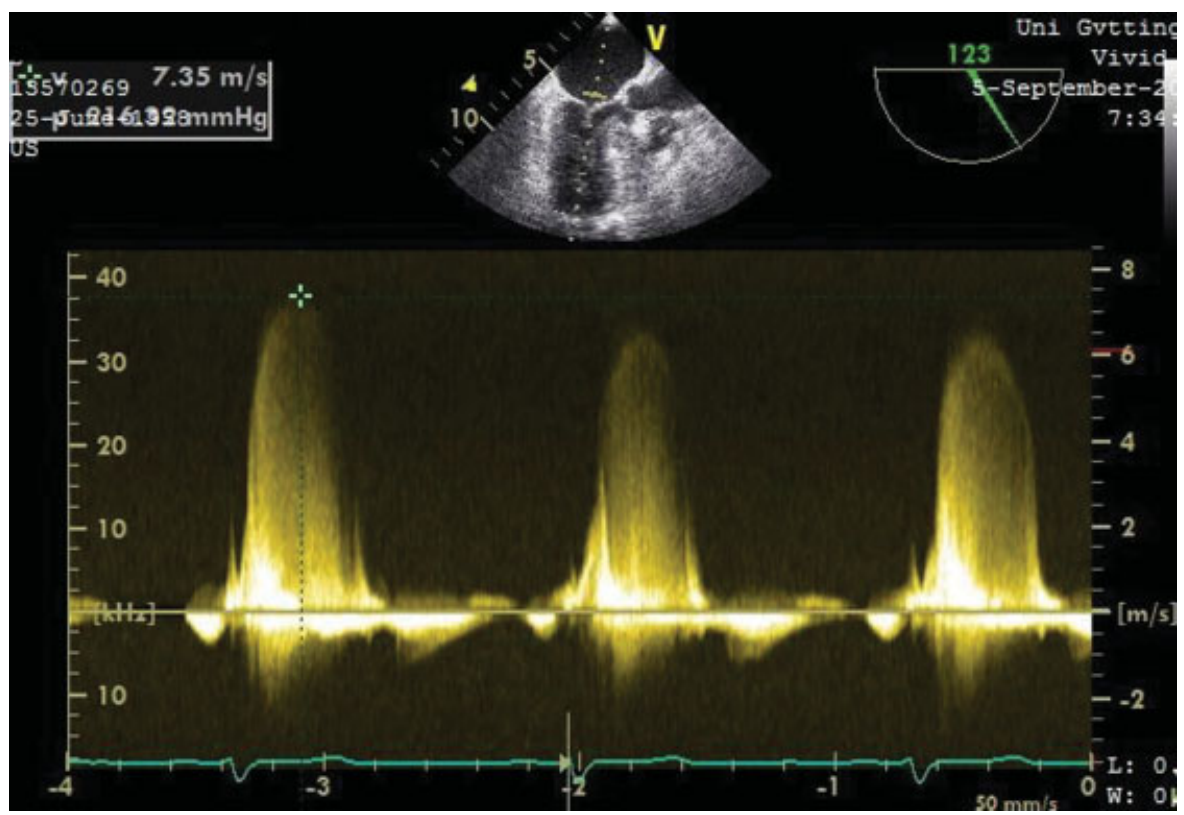

Fig. 1 Preoperative transesophageal echocardiogram showing severe obstruction with a $V_{\max }$ of $7.35 \mathrm{~m} / \mathrm{s}$ in the LVOT level. 
invasiveness and the reported good postintervention results. $^{5}$

Our patient underwent ASA with a good initial result directly after the intervention, in terms of reduction in pressure gradients. Despite these notably improved early measurements, recurrence of obstruction with clinical deterioration of the patient occurred after only 1 year. Moreover, intraoperative transesophageal echocardiography revealed even worse measurements. The surgeons were surprised by the finding of a subaortic membrane with firm trabeculae on the midventricular LVOT and by the fixed insertion of a part of the posterior papillary muscle within the anterior mitral valve leaflet. After correction of these findings and performance of extended myectomy, the obstruction of the outflow tract was significantly reduced. Because of the extended procedure, the need for permanent pacemaker was inevitable.

Results of ASA are reported to be very good, with a success rate of approximately $80 \%$. The relatively high risk of complete AV-block after such an ablation in former times has been allegedly fallen to $10 \%$ by using temporary pacemaker implantation during the intervention and by reduction in the amount of injected alcohol. ${ }^{5}$ A question that arises is whether the reduced amount of alcohol correlates with a reduced benefit in terms of ventricular relief and long-term outcome. Currently published data indicate that surgery is superior to ablation in reducing resting and provoked gradients, whereas the overall procedural complication rates are comparable in both techniques. ${ }^{6}$

In our case, we consider that the extended form of HOCM had doomed ablation to fail, a fact that is supported by the early recurrence of obstruction. The existence of a subaortic membrane and the strong fixation of the papillary muscle on the mitral valve are not typical findings, but when they do exist, surgery should be preferred for avoiding possible complications of both techniques, such as right bundle branch block following regularly ASA and left anterior hemiblock after myectomy, rendering the need for pacemaker implantation essential, when turning into left bundle branch block in case of large resections being necessary.

The decision of whether surgery or percutaneous ablation should be in the first line of treatment remains controversial.
The design of a randomized trial with a large population may be a utopia.

According to ACCF/AHA (American College of Cardiology Foundation/American Heart Association) guidelines for HCM, surgical septal myectomy, when performed in experienced centers, can be beneficial and is thus the first consideration for the majority of eligible patients with HCM with severe drug-refractory symptoms and LVOT obstruction (Class IIa, LOE B). $^{7}$

\section{References}

1 Richardson P, McKenna W, Bristow M, et al. Report of the 1995 World Health Organization/International Society and Federation of Cardiology Task Force on the Definition and Classification of cardiomyopathies. Circulation 1996;93(5):841-842

2 Maron BJ, Braunwald E. Evolution of hypertrophic cardiomyopathy to a contemporary treatable disease. Circulation 2012;126(13): $1640-1644$

3 Sigwart U. Non-surgical myocardial reduction for hypertrophic obstructive cardiomyopathy. Lancet 1995;346(8969):211-214

4 Schoendube FA, Klues HG, Reith S, Flachskampf FA, Hanrath P, Messmer BJ. Long-term clinical and echocardiographic follow-up after surgical correction of hypertrophic obstructive cardiomyopathy with extended myectomy and reconstruction of the subvalvular mitral apparatus. Circulation 1995;92(9, Suppl)II122-II127

5 Hess OM, Sigwart U. New treatment strategies for hypertrophic obstructive cardiomyopathy: alcohol ablation of the septum: the new gold standard? J Am Coll Cardiol 2004;44(10):2054-2055

6 Dearani JA, Ommen SR, Gersh BJ, Schaff HV, Danielson GK. Surgery insight: septal myectomy for obstructive hypertrophic cardiomyopathy-the Mayo Clinic experience. Nat Clin Pract Cardiovasc Med 2007;4(9):503-512

7 Gersh BJ, Maron BJ, Bonow RO, et al; American College of Cardiology Foundation/American Heart Association Task Force on Practice Guidelines; American Association for Thoracic Surgery; American Society of Echocardiography; American Society of Nuclear Cardiology; Heart Failure Society of America; Heart Rhythm Society; Society for Cardiovascular Angiography and Interventions; Society of Thoracic Surgeons. 2011 ACCF/AHA guideline for the diagnosis and treatment of hypertrophic cardiomyopathy: a report of the American College of Cardiology Foundation/American Heart Association Task Force on Practice Guidelines. Circulation 2011;124(24):e783-e831 